\title{
The B1 defective type of bifurcated right upper lobe bronchus
}

\author{
Min Zhang ${ }^{1 \#}$, Ning Mao ${ }^{2 \#}$, Si-Hang Wang ${ }^{3}$, Qing-Chen Wu ${ }^{1}$, Ming-Jian Ge ${ }^{1}$ \\ ${ }^{1}$ Department of Cardiothoracic Surgery, The First Affiliated Hospital of Chongqing Medical University, Chongqing 400016, China; ${ }^{2}$ Department \\ of Cardiothoracic Surgery, Yongchuan Hospital of Chongqing Medical University, Chongqing 402160, China; ${ }^{3}$ Department of Radiology, The First \\ Affiliated Hospital of Chongqing Medical University, Chongqing 400016, China \\ Contributions: (I) Conception and design: M Zhang; (II) Administrative support: N Mao; (III) Provision of study materials or patients: SH Wang; (IV) \\ Collection and assembly of data: QC Wu; (V) Data analysis and interpretation: MJ Ge; (VI) Manuscript writing: All authors; (VII) Final approval of \\ manuscript: All authors. \\ \#These authors contributed equally to this work. \\ Correspondence to: Qing-Chen Wu. Department of Cardiothoracic Surgery, The First Affiliated Hospital of Chongqing Medical University, \\ Chongqing 400016, China. Email: wqc6@hotmail.com; Ming-Jian Ge. Department of Cardiothoracic Surgery, The First Affiliated Hospital of \\ Chongqing Medical University, Chongqing 400016, China. Email: doctor1984@sina.com.
}

Background: As the more application of high-resolution computed tomography (HRCT), a great number of ground glass opacity (GGO) is identified. Video-assisted thoracoscopic surgery (VATS) segmentectomy is technically more difficult than lobectomy because of the anatomical complexity. Three-dimensional computed tomography bronchography angiography (3D-CTBA) is a powerful tool for thoracic surgeons to analyze pulmonary anatomy, allowing a better understanding of the pulmonary anatomy in each patient. Here we encountered seven cases of bifurcated right upper bronchus (B1 defective). The variation in vascular pattern of these patients is analyzed.

Methods: Between October 2018 and December 2018, a consecutive 162 patients with pulmonary lesions were admitted and underwent 3D-CTB prior to surgery. A total of seven cases of bifurcated right upper bronchus (B1 Defective) were identified. Then 3D-CTBA reconstruction was performed by Syngo MultiModality Workplace (Software: Syngo MMWP, Version: VF40A). Radiology colleagues processed all 3D images and thoracic surgeons confirmed the validity of all reconstructions.

Results: The mean age of the seven patients ( 3 females and 4 males) is 54 years. According to the branches of artery, they are divided into two types: "Tr. sup + A. asc" (2/7, 28.6\%) and "Tr. sup + Tr. inf + A. asc" (5/7, $71.4 \%$ ). According to the branches of A2 asc, another two types can be divided: type A, two branches of A2a asc $+\mathrm{A} 2 \mathrm{~b}$ asc $(4 / 7,57.1 \%)$ and type B, only one branch of A2b asc (3/7, 42.9\%). Types can also be divided according to the branches of A2 rec: A, no A2 rec (4/7, 57.1\%); B, one branch of A2a rec (2/7, 28.6\%); C, two branches of A2a rec + A2b rec (1/7, 14.3\%). According to the origins of A3, three types can be divided: A, A3 origins from Tr. inf (1/7, 14.3\%); B, A3 origins from Tr. sup (2/7, 28.6\%); C, A3 origins from both Tr. sup and Tr. inf (4/7, 57.1\%).

Conclusions: The "defective B1" type of bifurcated right upper lobe (RUL) bronchus is relatively rare. A pre-operative understanding of its anatomical features, especially the vascular variation patterns, may be helpful for completing a satisfactory segmentectomy. 3D-CTBA is a powerful tool, allowing a better understanding of the pulmonary anatomy in each patient before and during surgical procedures.

Keywords: Bifurcated; right upper lobe (RUL); variation; three-dimensional computed tomography bronchography angiography (3D-CTBA)

Submitted May 10, 2019. Accepted for publication Aug 23, 2019.

doi: $10.21037 /$ jtd.2019.09.64

View this article at: http://dx.doi.org/10.21037/jtd.2019.09.64

(c) Journal of Thoracic Disease. All rights reserved. 


\section{Introduction}

In the past two decades, the video-assisted thoracoscopic surgery (VATS) method has been increasingly performed. Lobectomy by VATS has been proven to be safe and effective in the treatment of early-stage NSCLC. Nowadays, as high-resolution computed tomography (HRCT) is commonly used for healthy examinations in countries like China, more ground glass opacities (GGOs) are identified. For these lesions, especially for those with diameters of less than $2 \mathrm{~cm}$ and consolidation tumor ratio (CTR) less than $50 \%$, lobectomy means more sacrifice of lung volume and less protection of lung function. These disadvantages have led to a new interest in the thoracoscopic approach to anatomic segmentectomy. Several studies have demonstrated similar outcomes between a VATS lobectomy and segmentectomy (1-5). However, segmentectomy is technically more difficult than lobectomy because of the anatomical complexity (both vascular and bronchial structures can vary greatly at the segmental level). A thorough preoperative analysis of the anatomic details and features is of great importance for more precise surgery. It is challenging to identify all the details from conventional two-dimensional (2D) CT images, especially for those with anatomic anomalies. Fortunately, advances in computed tomography (CT) and the volume-rendering reconstruction technique have allowed for the reconstruction of threedimensional (3D) images (6). 3D-CT bronchography (3D-CTB) and 3D-CT bronchography angiography (3D-CTBA) imaging are powerful tools for thoracic surgeons to determine pulmonary anatomy more intuitively compared with conventional CT images, allowing for a better understanding of the pulmonary anatomy in each patient before and during surgical procedures.

Among all of the lobes, the right upper lobes (RUL), because of a relatively high incidence of a tumor, undergoes more lobectomies and segmentectomies. Some studies have investigated its bronchovascular variations using 3D-CTBA (7). The right upper bronchus can be divided into three types (7): the trifurcated type $(44.1 \%)$, the bifurcated type $(29.3 \%)$ and the quadrivial type $(0.8 \%)$. The bifurcated type was further divided into three subtypes: the "B3 and B1 + 2" type, the "defective B1" type and the "defective B2" type. For the "defective B1" type, the B1 was replaced by BX1a and BX1b, which joins with B2 and B3 separately (B2 + BX1a and B3 + BX1b, Figure 1). We encountered 7 cases of the $\mathrm{B} 1$ defective type of bifurcated right upper bronchus. This bronchial variation can be

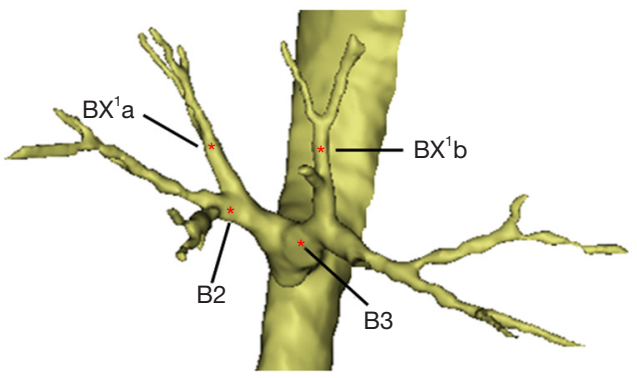

Figure 1 The defective B1 type branching of the RUL bronchus (Mimics Medical, serial number: 92C5-18A9-46A0-3C5C). RUL, right upper lobe.

identified even in HRCT, but the vascular variations are always accompanied with bronchial variations and complicated to identify. Considerable attention needs to be paid to the pre-operative evaluations for this kind of patient. This report describes the 7 cases of bifurcated types, analyzing the vascular variations using $3 \mathrm{D}-\mathrm{CTBA}$, providing more accurate information for a pre-operative evaluation.

\section{Methods}

\section{Reconstruction of 3D-CTBA}

CTPA and CTPV by SOMATOM Definition Flash dualsource computed tomography were performed for each patient. 3D-CTBA reconstruction was analyzed by Syngo MultiModality Workplace (software: Syngo MMWP, Version: VF40A). Radiology colleagues processed all 3D images, and thoracic surgeons confirmed the validity of all reconstructions. Sub-segmental vessels/branches less than $5 \mathrm{~mm}$ in diameter prove difficult to visualize adequately on 3D-CTBA. However, as long as these small structures can be identified from the HRCT, they can also be reconstructed using some specific steps manually in postprocessing.

\section{Patient characters}

Between October 2018 and December 2018, all patients with pulmonary lesions underwent 3D-CTB before surgery, and 162 patients were finally included. A total of 7 cases of the bifurcated right upper bronchus (B1 defective) were identified. The incidence rate was $4.3 \%$. Then, $3 \mathrm{D}$-CTBA reconstruction was performed for these patients. The nomenclature used to describe the structures is that of 
A
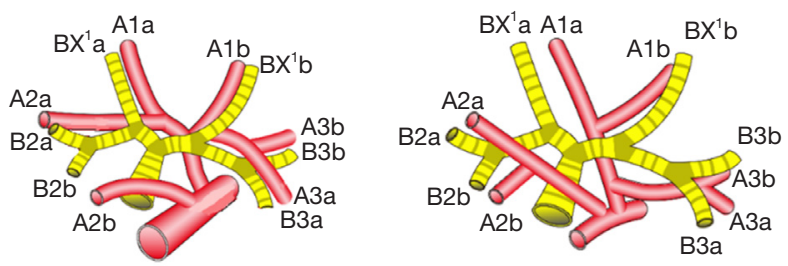

B
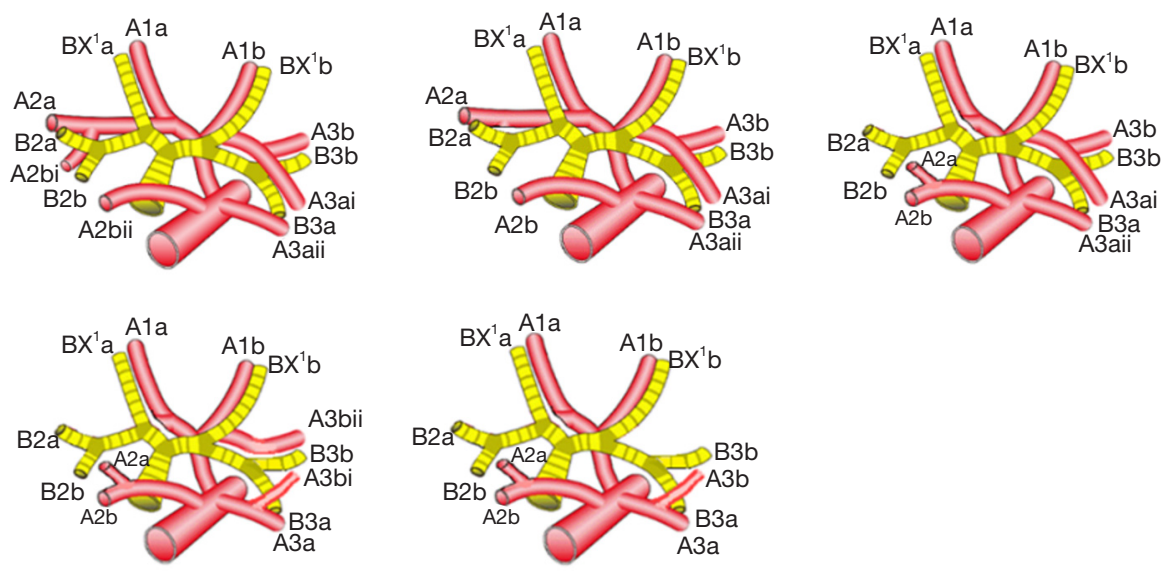

Figure 2 Simplified models of RUL artery and bronchus. (A) The "Tr. sup + A. asc" type; (B) the "Tr. sup + Tr. inf + A. asc" type. RUL, right upper lobe.

Shimizu (8). This retrospective study was approved by the ethics committee. Informed consent was obtained from each involved patient.

\section{Variation in bronchovascular pattern}

The classical branching of the RUL vein as follows: (I) the anterior vein $(\mathrm{V}$. ant) which originates from the $\mathrm{V} 1 \mathrm{~b}$, descends anteriorly to the upper lobe bronchus, and drains into the main RUL vein from the mediastinal side; (II) central vein (V. cent), which originates from the V2a, descends through the center of the upper lobe, and drains into the main RUL vein from the interlobar side. This "anterior + central" form was divided into two subtypes (Iab and $\mathrm{Ib}$ ). In the Iab type, V1a and V1b merge into V. ant. In the Ib type, V1a is termed VX1a and drains into V. cent., whereas V. ant originates only from V1b. Other forms of the RUL vein, like the "anterior" form and "central" form (8), do not appear in our 7 cases.

Branching of the RUL artery was defined as trunk superior (Tr. sup), trunk inferior (Tr. inf), ascending artery (A. asc) and recurrent artery (A. rec) (7). Tr. sup is the first branch of the right pulmonary artery. Tr. inf is the second branch of the right pulmonary artery; between Tr.
Sup and the first artery of middle lobe, Tr. inf arises from the mediastinal portion of the artery. A. asc arises from the interlobar portion of the right pulmonary artery and generally branches from the distal region of the first middle lobe artery. A. rec branches from the Tr. sup. It crosses behind the BX1a to supply the S2a or S2a + b.

\section{Results}

The mean age of the 7 patients ( 3 females and 4 males) was 54 years. According to the branches of the artery, there were two types defined: “Tr. sup + A. asc" $(2 / 7,28.6 \%$, Figure $2 A)$ and "Tr. sup + Tr. inf + A. asc" (5/7, 71.4\%, Figure $2 B$ ). According to the branches of A2 asc, two other types can be defined: type A, two branches of A2a asc + A2b asc $(4 / 7,57.1 \%$, Figure $3 A)$; type B, only one branch of A2b asc $(3 / 7,42.9 \%$, Figure $3 B)$. Types can also be defined according to the branches of $\mathrm{A} 2$ rec: $\mathrm{A}$, no $\mathrm{A} 2 \mathrm{rec}$ $(4 / 7,57.1 \%$, Figure $4 A)$; B, one branch of A2a rec $(2 / 7$, $28.6 \%$, Figure $4 B)$; C, two branches of A2a rec $+\mathrm{A} 2 \mathrm{~b}$ rec $(1 / 7,14.3 \%$, Figure $4 C)$. According to the origins of A3, three types can be defined: A, A3 origins from Tr. inf (1/7, $14.3 \%$, Figure $5 A)$; B, A3 origins from Tr. sup (2/7, 28.6\%, Figure 5B); C, A3 origins from both Tr. sup and Tr. inf (4/7, 

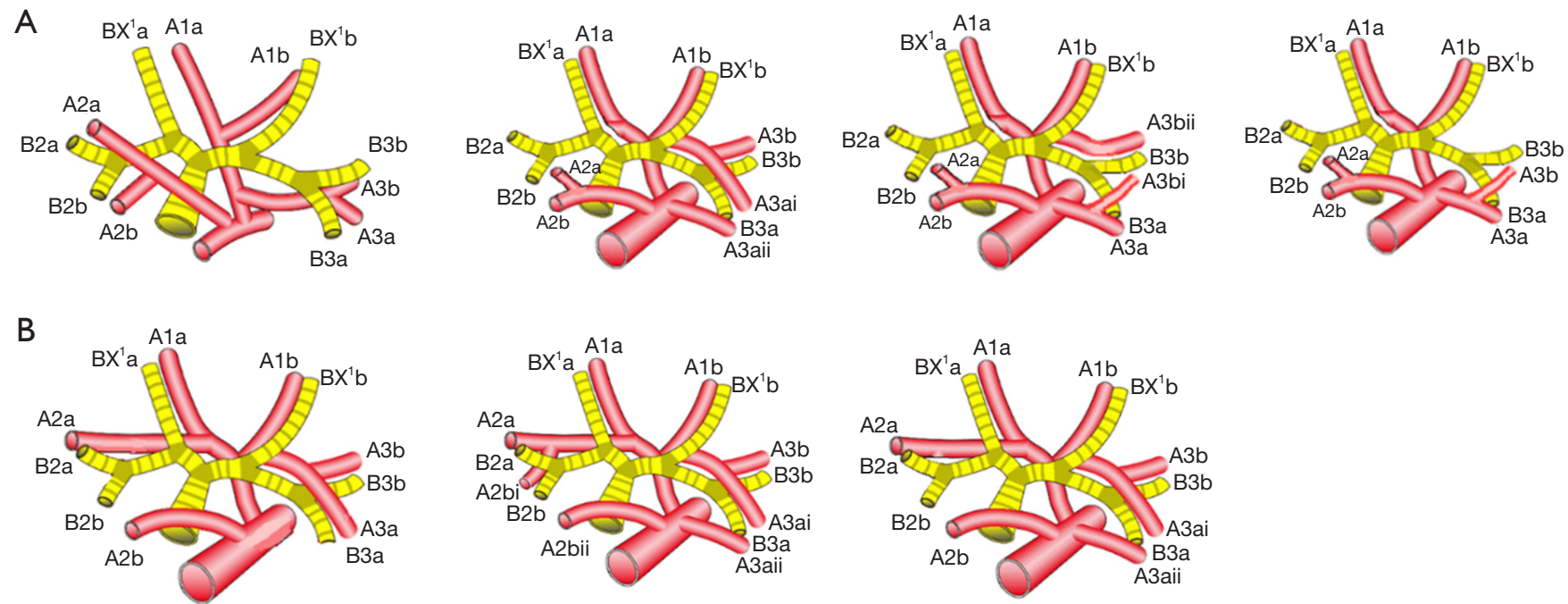

Figure 3 Branch patterns of A2 asc. (A) Two branches of A2a asc + A2b asc; (B) one branch of A2b asc.
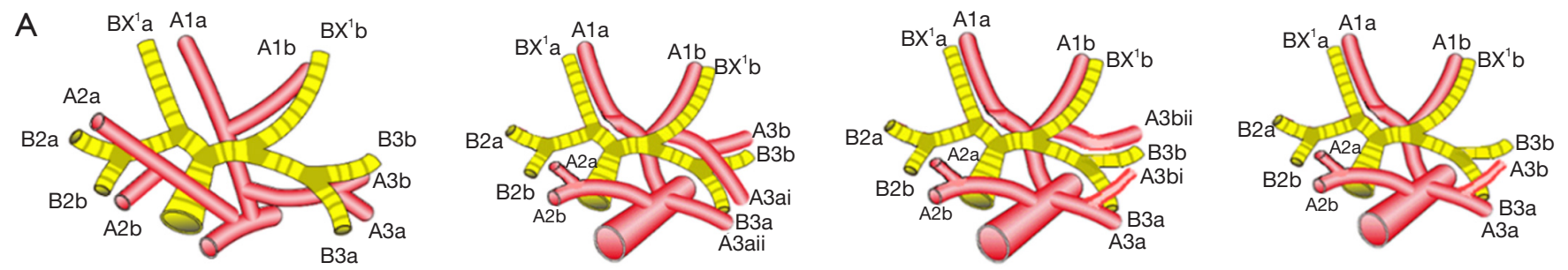

B
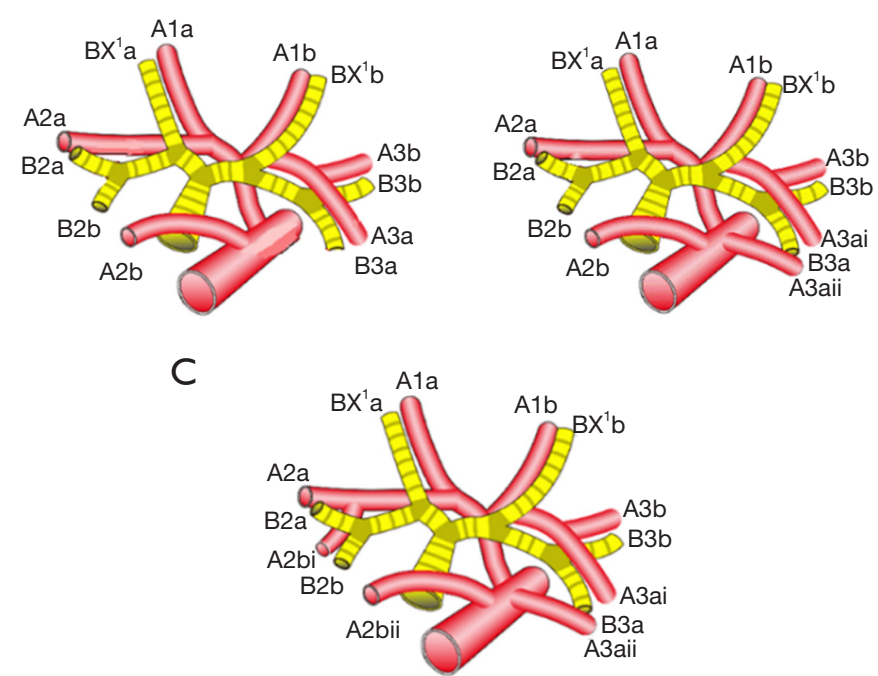

Figure 4 Branch patterns of A2 rec. (A) No A2 rec; (B) one branch of A2a rec; (C) two branches of A2a rec + A2b rec. 
A
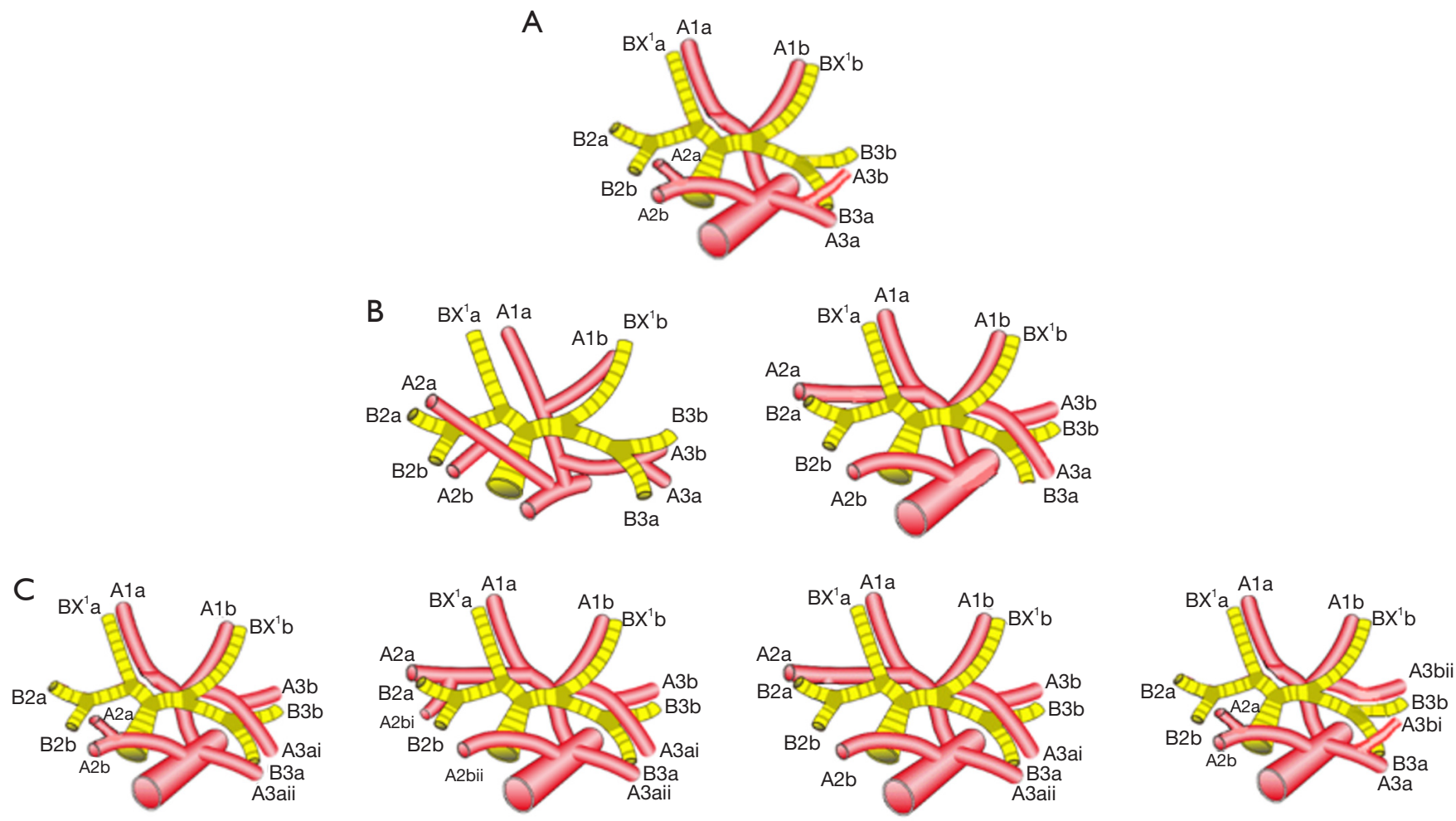

Figure 5 Origins of A3. (A) Origins from Tr. inf (A); (B) origins from Tr. sup (B); (C) origins from both Tr. sup and Tr. inf (C).

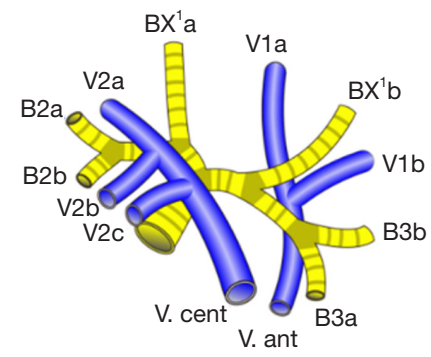

Type lab

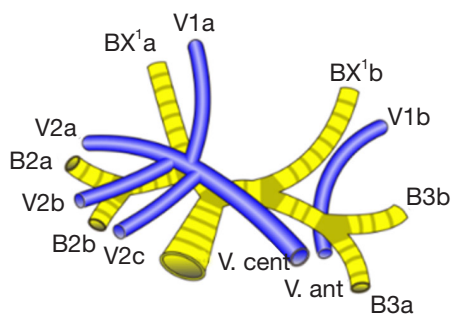

Type Ib

Figure 6 Simplified models of RUL vein and bronchus. RUL, right upper lobe.

\section{$57.1 \%$, Figure 5C).}

For the vein, there are two types: "Iab" type (2/7, 28.6\%) and "Ib" type (5/7, 71.4\%) (Figure 6).

\section{Discussion}

As the application of 3D-CTBA, the bifurcated right upper bronchus has become more and more familiar to surgeons. Here we report one particular type of bifurcated right upper bronchus, the "defective B1" type, which was first reported by
Nagashima et al. in 2015 (7). The incidence is 4.3\% in our study, which is similar to that of Nagashima et al. (4.9\%) (7). However, the vascular variation of this type has not been reported in literature. According to our experiences, knowing only the bronchial variation is far from enough to perform a good segmentectomy for this kind of patient. The vascular variation is always accompanied and complicated, requiring a thorough pre-operative evaluation.

Two artery branching types, using the nomenclature of Shimizu, appeared in our study: the "Tr. sup + A. asc" 
type (type A, 28.6\%) and "Tr. sup + Tr. inf + A. asc" type (type B, 71.4\%). Other types, like "Tr. sup" type and "Tr. sup + Tr. inf" type, did not appear (7). It seems that A. asc persistently exists in the "defective B1" type of the bifurcated right upper bronchus. The branch pattern of A. asc has its clinical significance in practice. For example, for an estimated segmentectomy of $\mathrm{S} 2 \mathrm{~b}$, it is important to know pre-operatively whether the $\mathrm{A} 2$ asc is unique or has two branches. If it is unique (Figure $3 B$ ), the cut of A2b may be enough. If it has two branches of A2a and A2b (Figure 3A), a mistaken cut at the trunk of $\mathrm{A} 2$ will result in an enlarged intersegmental plane. The branching pattern of A. rec also has its clinical significance. For example, for an estimated segmentectomy of S2a, it is also important to know preoperatively the blood supply of S2a: whether it is from A2 asc (Figure 4A), A2 rec (one branch) (Figure 4B) or A2a rec (two branches) (Figure $4 C$ ). Inadequately cutting the artery will result in a narrowed inter-segmental plane. For an estimated segmentectomy of $\mathrm{S} 3$, it is also important to know pre-operatively whether the blood supply of S3 is from Tr. inf, Tr. sup, or both, because inadequate cutting of the artery will result in a narrowed intersegmental plane. For the vein, there were only two types found in our study: "Iab" $(2 / 7,28.6 \%)$ and "Ib" (5/7, 71.4\%) (Figure 6). According to the principles of the inflation-deflation method, adequate occlusions of the artery (or vein), are companied by accurate recognition of the bronchus, which is sufficient for a satisfactory presentation of the intersegmental plane. In our practice, we have also recognized that it is not necessary to distinguish each branch of veins, as long as the artery and bronchus are correctly dealt with.

\section{Conclusions}

The "defective B1" type of bifurcated RUL bronchus is relatively new to surgeons. A pre-operative understanding of its anatomical features by 3D-CTBA, especially the vascular variation patterns, may help complete a satisfactory segmentectomy of this lobe.

\section{Acknowledgments}

None.

\section{Footnote}

Conflicts of Interest: The authors have no conflicts of interest to declare.
Ethical Statement: The authors are accountable for all aspects of the work in ensuring that questions related to the accuracy or integrity of any part of the work are appropriately investigated and resolved. This retrospective study was approved by the ethics committee. Informed consent was obtained from each involved patient.

\section{References}

1. Yamashita S, Tokuishi K, Anami K, et al. Thoracoscopic segmentectomy for T1 classification of non-small cell lung cancer: a single center experience. Eur J Cardiothorac Surg 2012;42:83-8.

2. Zhong C, Fang W, Mao T, et al. Comparison of thoracoscopic segmentectomy and thoracoscopic lobectomy for small-sized stage IA lung cancer. Ann Thorac Surg 2012;94:362-7.

3. Zhang L, Ma W, Li Y, et al. Comparative study of the anatomic segmentectomy versus lobectomy for clinical stage I A peripheral lung cancer by video assistant thoracoscopic surgery. J Cancer Res Ther 2013;9 Suppl 2:S106-9.

4. Zhao X, Qian L, Luo Q, et al. Segmentectomy as a safe and equally effective surgical option under complete videoassisted thoracic surgery for patients of stage I non-small cell lung cancer. J Cardiothorac Surg 2013;8:116.

5. Ren M, Meng Q, Zhou W, et al. Comparison of shortterm effect of thoracoscopic segmentectomy and thoracoscopic lobectomy for the solitary pulmonary nodule and early-stage lung cancer. Onco Targets Ther 2014;7:1343-7.

6. Rubin GD. 3-D imaging with MDCT. Eur J Radiol 2003;45 Suppl 1:S37-41.

7. Nagashima T, Shimizu K, Ohtaki Y, et al. An analysis of variations in the bronchovascular pattern of the right upper lobe using three-dimensional CT angiography and bronchography. Gen Thorac Cardiovasc Surg 2015;63:354-60.

8. Shimizu K, Nagashima T, Ohtaki Y, et al. Analysis of the variation pattern in right upper pulmonary veins and establishment of simplified vein models for anatomical segmentectomy. Gen Thorac Cardiovasc Surg 2016;64:604-11.

Cite this article as: Zhang M, Mao N, Wang SH, Wu QC, Ge MJ. The B1 defective type of bifurcated right upper lobe bronchus. J Thorac Dis 2019;11(10):4218-4223. doi: 10.21037/ jtd.2019.09.64 